\title{
Evaluación de desempeño de un controlador PI neurodifuso para una unidad turbogás
}

\section{Luz Yazmin Villagrán-Villegas ${ }^{1}$ \\ Miguel Patiño-Ortiz ${ }^{1}$ \\ Miguel Ángel Martínez-Cruz ${ }^{1}$ \\ Ana Alejandra Patiño-Ortiz ${ }^{1}$ \\ Tiberio Venegas-Trujillo² \\ Luis Alberto Contreras-Aguilar ${ }^{2}$}

\author{
${ }^{1}$ Instituto Politécnico Nacional \\ Escuela Superior de Ingeniería Mecánica y Eléctrica, \\ Sección de Estudios de Posgrado e Investigación, \\ Unidad Profesional Adolfo López Mateos "Zacatenco" \\ Edificio 5 2do. Piso, Col. Lindavista, C.P. 07300, \\ Ciudad de México. \\ MEXICO

\section{'Universidad de Colima \\ Facultad de Ingeniería Mecánica Eléctrica \\ Av. Universidad No. 333, Las Víboras, \\ C.P. 28040, Colima. \\ MEXICO}

correo electrónico (email): yazvillagranv@gmail.com

Recibido 03-09-2019, aceptado 21-11-2019.

\section{Resumen}

Las turbinas de gas son utilizadas como impulsores primarios en la generación de energía eléctrica. El desempeño de una unidad de turbo gas es dependiente de la unidad de control que tenga, ya que en ella radica gran parte de la optimización en la ejecución del sistema. En este trabajo se presentan los resultados de la evaluación de la estabilidad del controlador en el desempeño de un modelo de un controlador neurodifuso para una unidad turbogás, lo anterior como un primer paso para su aplicación en un proceso real. El diseño del controlador neurodifuso se basa en un controlador PI digital del tipo velocidad. El análisis y evaluación del controlador se hacen mediante simulaciones en tiempo discreto con un sistema de primer orden con retardo. Las pruebas incluyen variaciones de los parámetros del proceso: ganancia, retardo y constante de tiempo. Se evalúa el efecto de perturbaciones determinísticas y estocásticas en la señal de control, la variable mani- pulada y la variable controlada. Los resultados obtenidos demuestran la factibilidad de utilizar un controlador neurodifuso, integrado en equipos digitales, para aplicaciones reales.

Palabras clave: sistema neurodifuso, control neurodifuso, control digital, análisis de desempeño.

\section{Abstract \\ (Performance Evaluation of a PI Neuro-Fuzzy controller for a Turbogas Unit)}

Gas turbines are used as primary impellers in the generation of electrical energy. The performance of a turbo gas unit is dependent on the control unit that it has, it is significant in optimization in the system execution. This paper shows the results of the evaluation of robustness in the performance of a model of a neuro -fuzzy controller for a turbogas unit, it is a first step for application in the real process. The design of the neuro-fuzzy controller is based on a digital PI controller of the speed type. The analysis and evaluation of the controller are done by simulations in discrete time with a first order system with delay. The tests include variations of the process parameters: gain, delay and time constant. The effect of deterministic and stochastic disturbances on the control signal, the manipulated variable and the controlled variable is evaluated. The results obtained demonstrate the feasibility of using a neuro-fuzzy controller, integrated in digital equipment, for real applications.

Index terms: neuro-fuzzy system, neuro-fuzzy control, digital control, analisys of performance.

\section{Introducción}

La unidad turbogás es una máquina de combustión que puede convertir gas natural o cualquier otro combustible en energía mecánica, esta energía impulsa un generador que produce energía eléctrica [1], [2], [3]. Durante el desarrollo de los sistemas de control para centrales generadoras de la CFE [4], [5], se ha detectado la necesidad de mejorar las estrategias de control de las unidades turbogás que garanticen una operación más segura y rentable, ya que las unidades turbogás operan a temperaturas, presiones y velocidades relativamente más altas que las de cualquier otro tipo de unidad, necesitando mayores y más estrictos requerimientos para el sistema de control de estos parámetros de operación [6].

Actualmente, los sistemas de control de unidades turbogás están basados en algoritmos de control convencionales del 
tipo PI (Proporcional-Integral), y son usados para tareas de regulación, sin embargo, se debe analizar su uso para el control de velocidad durante el arranque o para el control de potencia generada en una unidad turbogás. El desempeño de una unidad puede ser afectado adversamente por la no linealidad de la dinámica del proceso, la cual cambia conforme al punto de operación, pero a pesar de ello, el uso de los controladores PI se ha prolongado por mucho tiempo debido a su sencillez y a que, estrictamente, no se requiere de un modelo matemático del proceso para su diseño y ajuste, razón por la cual no se haya generalizado la aplicación en estrategias de control moderno. Una alternativa para mejorar el desempeño de las unidades turbogás es el empleo de técnicas de Inteligencia Artificial (IA) para el control de procesos industriales [7], [8], [9], mejorando el control moderno y satisfaciendo requerimientos que no se pueden con el control PI convencional.

En el Instituto Nacional de Electricidad y Energías Limpias (Ex Instituto de Investigaciones Eléctricas) se rediseñó la programación del sistema de control para una unidad turbogás modelo W501 GE [10], se incorporó un controlador difuso de velocidad en tiempo real para el arranque de la turbina de gas [11], [12] y se trabajó en el control de la potencia generada [13], [14]. En estos casos, los resultados obtenidos fueron exitosos y constituyen una base sólida para la aplicación de controladores difusos en una unidad turbogás real.

Uno de los problemas fue la dificultad para obtener la base de reglas y los parámetros de los diversos elementos del sistema de inferencia difuso integrado en el controlador. Para solucionar la problemática y automatizar lo más posible el diseño del sistema de inferencia difuso, se desarrolló un sistema de sintonización basado en una red neuronal aplicado a un controlador PI convencional y a un controlador difuso [15], [16]. El conjunto formado por la red neuronal y el sistema de inferencia difuso es del tipo cooperativo, en donde ambos componentes son aplicaciones independientes, la red neuronal le comunica los resultados de aprendizaje al sistema difuso. Para mejorar el sistema neurodifuso cooperativo, se planteó el desarrollo de un controlador neurodifuso híbrido, en donde una red neuronal y un sistema de inferencia difuso se integran. En este tipo de sistemas híbridos la determinación de los parámetros del sistema de inferencia difuso se lleva a cabo de una manera directa y más simple, aprovechando algún algoritmo de aprendizaje neuronal.

En este trabajo se presenta un controlador neurodifuso digital basado en un algoritmo de control PI convencional del tipo velocidad. El diseño se realiza fuera de línea en forma automatizada en una computadora personal y puede ser implantado directamente en una plataforma digital de grado industrial para su aplicación a un proceso real. Se muestran los resulta- dos del análisis y evaluación de la estabilidad del controlador del desempeño del controlador. El análisis y evaluación se hacen mediante experimentos de simulación en tiempo discreto, aplicando el controlador a un sistema de primer orden con retardo. Las pruebas consisten en evaluar la respuesta del sistema ante variaciones en rango amplio de los parámetros del proceso, los cuales incluyen la ganancia, el retardo y la constante de tiempo. Se evalúa el efecto de perturbaciones determinísticas y estocásticas en la señal de control, la variable manipulada y la variable controlada. Los resultados obtenidos demuestran la factibilidad de utilizar un controlador híbrido neurodifuso en aplicaciones reales.

\section{Controlador neurodifuso}

El objetivo principal de la integración de las técnicas de sistemas difusos y redes neuronales en un sistema neurodifuso híbrido (SNDH) es para facilitar el diseño del sistema difuso, aplicando las técnicas de aprendizaje neuronal. En general, se busca determinar la estructura y/o los parámetros del sistema difuso. El aprendizaje estructural comprende el diseño de las reglas de inferencia, determinando el número de variables a tomar en cuenta, la partición del universo de discurso de cada variable, el número y la forma de las reglas. El ajuste de parámetros se refiere a la determinación de valores para los parámetros que definen las funciones de pertenencia, los conectivos lógicos difusos y los pesos de las reglas de inferencia. En este trabajo, el problema del diseño del controlador neurodifuso híbrido se formula como un problema de ajuste de parámetros en el que la estructura del SNDH se asume conocida, con base en los trabajos mencionados en la introducción. Entonces, se tiene un problema de aprendizaje supervisado, en el que dados el conjunto de patrones de datos de entrada-salida, a ser reproducidos por la red neuronal y la arquitectura de la red neuronal, se tienen que determinar los parámetros de la red neuronal mediante algún algoritmo de entrenamiento, o equivalentemente, los parámetros del SNDH asociado. Específicamente, se desea que el SNDH implemente el mapeo estático, $R^{2} \rightarrow R$, definido por un controlador PI digital convencional tipo velocidad; tomando como entradas el error $e$ y el cambio del error $\Delta e, \mathrm{y}$ como salida el cambio en la señal de control $\Delta u$ (véase Fig. 1). Debido a que ambos, un sistema de inferencia difuso o una red neuronal, tienen la propiedad de ser aproximadores universales [7]; el mapeo estático, definido por el controlador PI, puede ser aproximado con cualquier nivel de precisión deseado, y por tanto el controlador PI digital convencional puede ser sustituido directamente por el controlador neurodifuso híbrido equivalente, tal y como se muestra en la Fig. 2.

El diseño de un sistema de inferencia difuso no es una tarea trivial, la gran diversidad de formas en que los componentes 


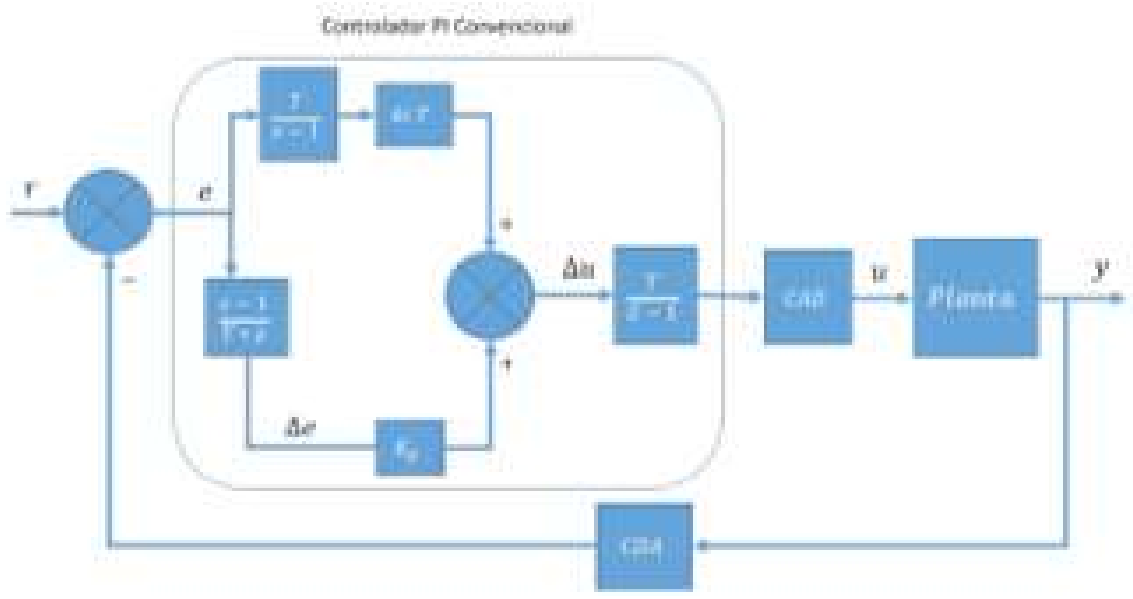

Fig. 1. Controlador PI digital convencional en configuración tipo velocidad.

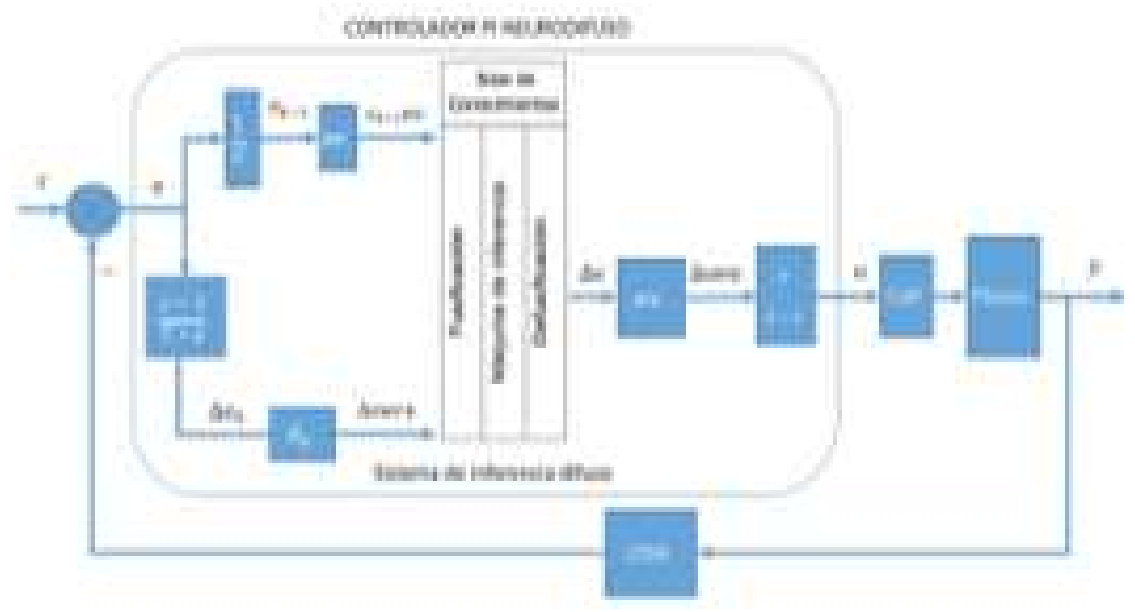

Fig. 2. Controlador PI neurodifuso híbrido.

de un sistema difuso pueden ser definidos e implementados, puede complicar innecesariamente el diseño del sistema. Algunas de las decisiones que deben tomarse incluyen la selección de los métodos de: fusificación, los conectivos lógicos, implicación, inferencia, composición y desfusificación. Adicionalmente, se debe considerar el crecimiento exponencial y geométrico del número de reglas en la base de conocimientos, debido al número de entradas y al número de funciones de pertenencia de cada una de las entradas, con lo cual se incrementa la carga de procesamiento y los requerimientos de memoria para la implementación, razones por las cuales, se deben utilizar solamente aquellos métodos que faciliten el diseño, sin comprometer el desempeño del controlador resultante. Una alternativa atractiva es utilizar sistemas de inferencia difuso del tipo Takagi-Sugeno-Kan (TSK), con funciones de pertenencia del tipo triangular en las entradas y singletones en la salida, y desfusificación por el método de centro de gravedad.

Para el caso del controlador digital neurodifuso híbrido, las reglas de inferencia del sistema TSK serán de la forma:

$$
\text { si } e \text { es } L_{e} \text { y } \Delta e \text { es } L \Delta e \text { entonces } \Delta u=k
$$

en donde $e$ es el error, $L_{e}$ un valor lingüístico del error, $\Delta e$ es el cambio del error, $L \Delta e$ un valor lingüístico de $\Delta e, \Delta u$ el cambio en la señal de control, y $k$ es una constante.

Dado el diseño inicial arbitrario de un sistema de inferencia difuso del tipo TSK, las funciones de pertenencia y las constantes en los consecuentes de las reglas pueden ser ajustados para reproducir un conjunto de patrones de entrada y salida, 


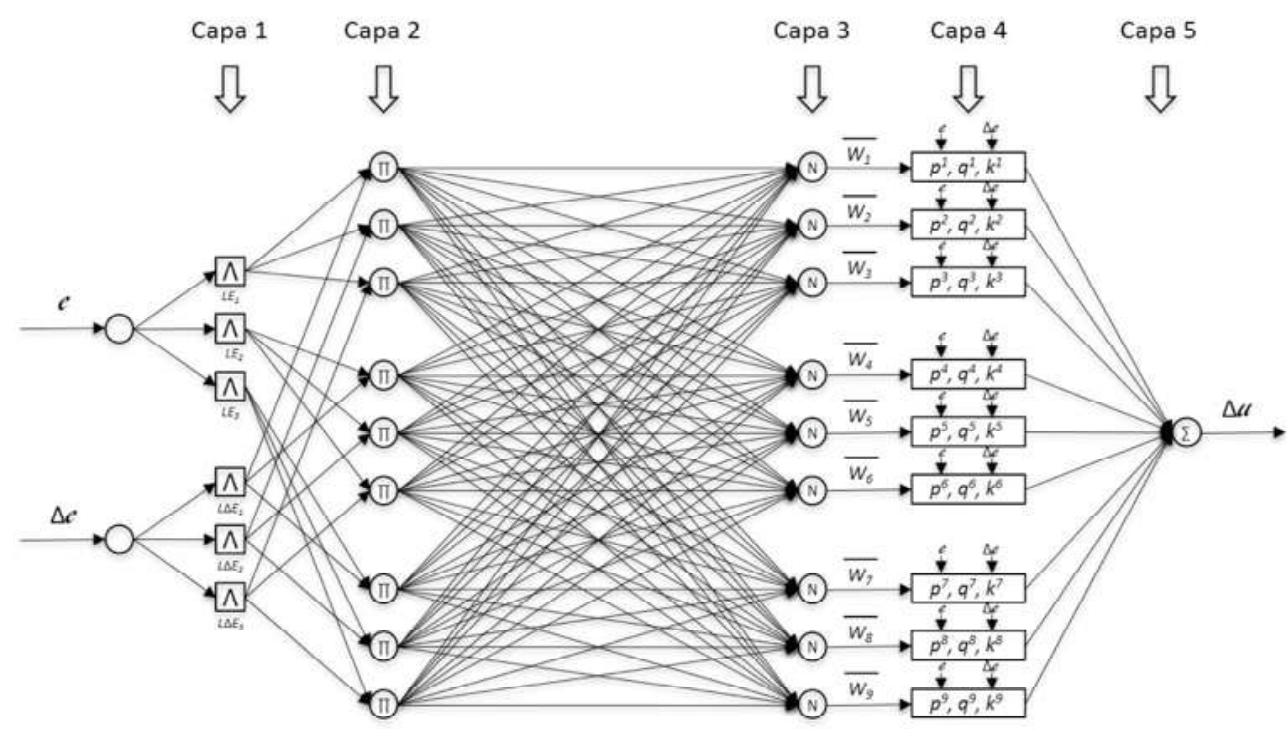

Fig. 3. Arquitectura del sistema de inferencia difuso del controlador como una red neuronal.

empleando el método [17]. En este método el sistema de inferencia difuso del tipo TSK del controlador digital neurodifuso es representado mediante una red neuronal tipo "feedforward" (véase Fig. 3). El proceso de aprendizaje, o equivalentemente de ajuste de las funciones de pertenencia y las constantes en los consecuentes de las reglas de inferencia, se desarrolla de la siguiente manera: Primero, un conjunto de patrones de datos de entrada y salida a ser usados como datos de entrenamiento, deben ser generados a partir del mapeo estático del controlador PI digital convencional. Otro conjunto de patrones puede ser usado como datos para chequeo después del entrenamiento. Segundo, la estructura inicial del sistema de inferencia difuso debe ser especificada mediante el número de funciones de pertenencia y la forma de estas para cada una de las entradas [18], [19]. Finalmente, el proceso de aprendizaje se ejecuta utilizando el conjunto de patrones de datos de entrenamiento para generar las reglas de inferencia, ajustar las funciones de pertenencia y determinar los parámetros de los consecuentes. El sistema de inferencia obtenido es verificado con el conjunto de patrones de datos para el chequeo.

En este trabajo, ambos conjuntos de patrones de datos de entrada y salida, para el entrenamiento y para el chequeo, se obtienen a partir del mapeo estático (véase Fig. 4), definido por la relación constitutiva del algoritmo de control PI digital tipo velocidad:

$$
\Delta u=K_{i} \Delta e+K_{p} c
$$

en donde $e$ es la señal de error, $\Delta e$ es el cambio del error, $\Delta u$ es el cambio en la señal de control, $K_{p}$ es la constante de ganan- cia proporcional, y $K_{i}$ es la constante de ganancia integral. Los valores de $K p$ y $K_{i}$ fueron determinados en dos pasos: primero, se aplicó el método de Ziegler-Nichols para obtener valores iniciales a partir de la respuesta del sistema a un cambio en escalón de la señal de referencia. Segundo, se realizó un ajuste fino de estos valores empleando una rutina de optimización, usando un algoritmo de mínimos cuadrados no lineales para minimizar el índice de desempeño IAE (integral del valor absoluto del error):

$$
I A E=\int_{0}^{t}|r-y| d \lambda
$$

El sistema TSK obtenido es un sistema de dos entradas y una salida, en el que las funciones de pertenencia para la fusificación de la entrada correspondiente a la señal del error son mostradas en la Fig. 5, mientras que las funciones de pertenencia de la entrada del cambio en el error se muestran en la Fig. 6. Los valores de los parámetros de los consecuentes para cada una de las reglas se muestran en la Tabla 1; el sistema TSK tiene reglas con consecuentes constantes.

\section{Análisis de desempeño}

Para la realización de los experimentos de simulación, que permitieron analizar el desempeño del controlador digital neurodifuso, se programó un lazo de control simple en el ambiente Matlab/Simulink, en una plataforma de computadora personal. El proceso para controlar es simulado con un modelo de primer orden con retardo: 


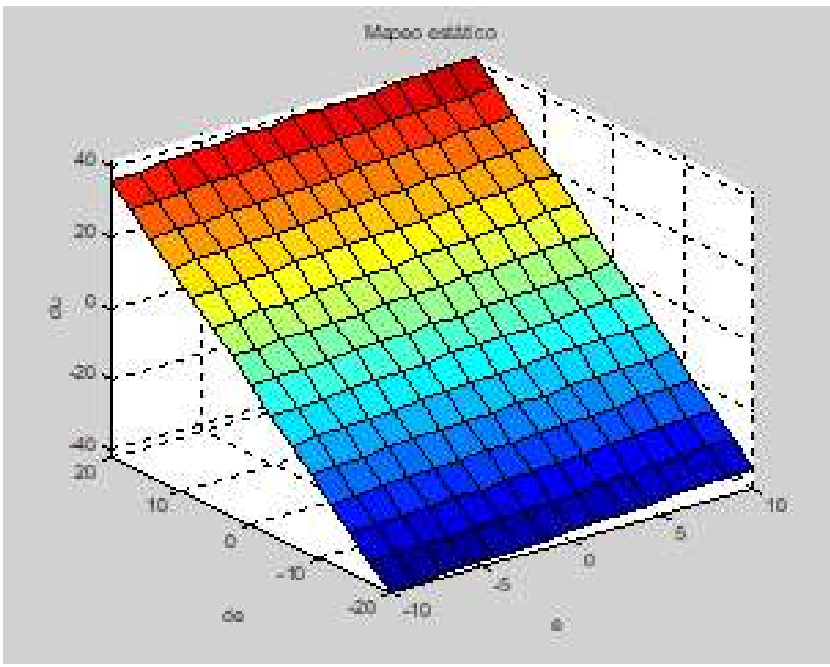

Fig. 4. Mapeo estático definido por el controlador PI digital convencional.

$$
G(s)=\frac{K_{d c} e^{-t_{d} s}}{\tau s+1}
$$

en donde $K_{d c}$ es la ganancia en estado estable, $t_{d}$ es el retardo en el tiempo, $\tau$ es la constante de tiempo, y $s$ es la variable de Laplace. Los valores nominales de estos parámetros son $K_{d c}=2 ; t_{d}=0.4 \mathrm{~s} ; \tau=4.0 \mathrm{~s}$.

Para determinar las características de desempeño del controlador digital neurodifuso se llevaron a cabo varias pruebas en dos conjuntos. En el primer conjunto se evaluó la estabilidad del controlador neurodifuso ante cambios en los valores de los parámetros de la planta. En el segundo conjunto se sometió al lazo de control a perturbaciones estocásticas y determinísticas en las señales más importantes. En ambos

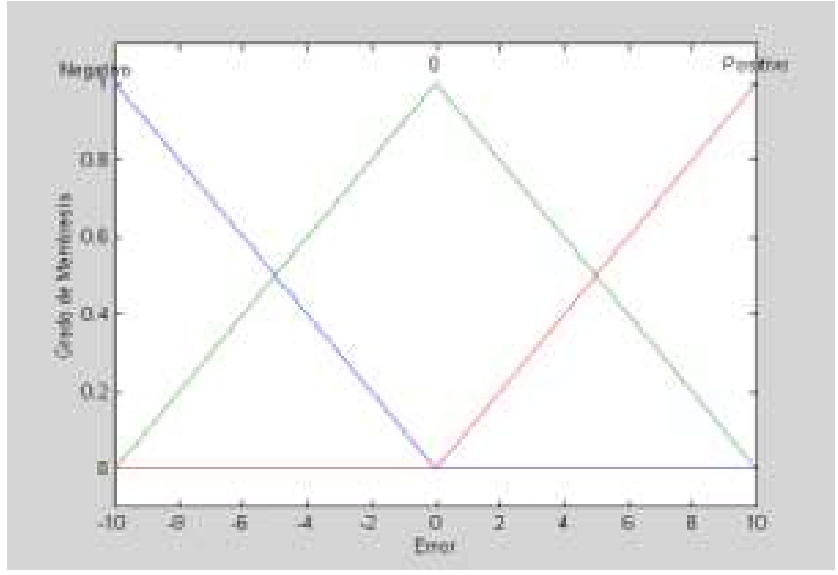

Fig. 5. Funciones de pertenencia de la señal de error.

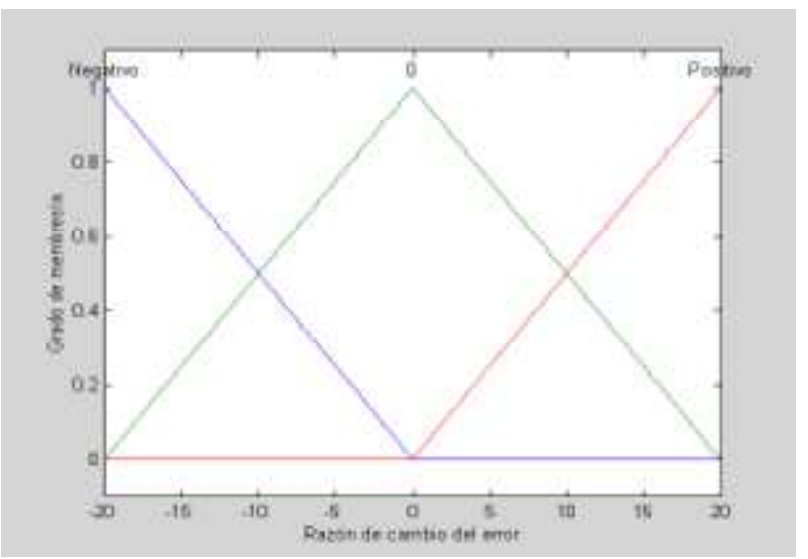

Fig. 6. Funciones de pertenencia de la señal de cambio del error.

conjuntos de pruebas se evaluaron varios índices de desempeño durante la respuesta a un cambio en escalón en la señal de referencia. En este artículo, sólo se presentan los resultados obtenidos con el índice IAE.

Las figuras 7, 8, 9 y 10 muestran la respuesta del sistema utilizando el controlador PI digital convencional (trazo conti-

Tabla 1. Reglas del controlador neurodifuso.

\begin{tabular}{ccccc} 
& \multicolumn{4}{c}{$e$} \\
\hline & $\Delta u$ & negativo & cero & positivo \\
$\Delta e$ & positivo & $k_{1}$ & $k_{2}$ & $k_{3}$ \\
& cero & $k_{4}$ & $k_{5}$ & $k_{6}$ \\
& negativo & $k_{7}$ & $k_{8}$ & $k_{9}$ \\
\hline
\end{tabular}

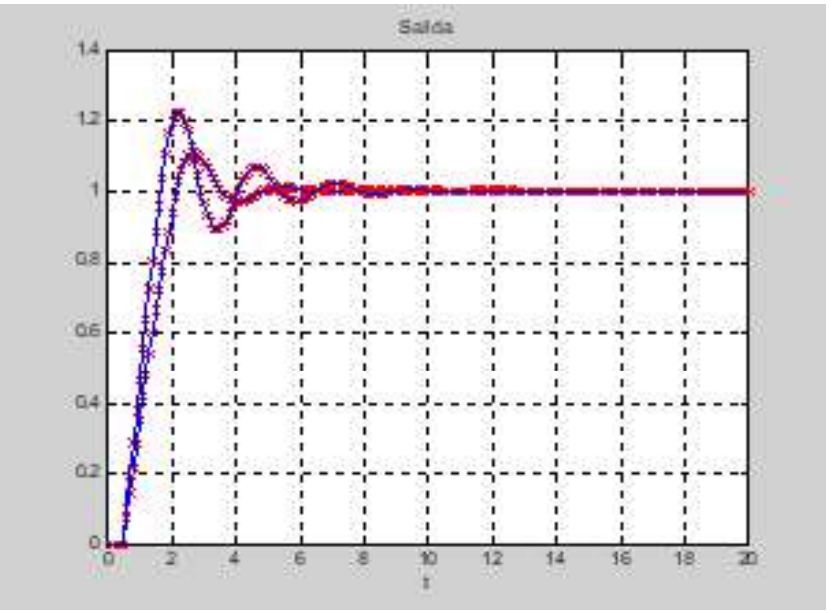

Fig. 7. Respuesta ante variaciones en la ganancia. 


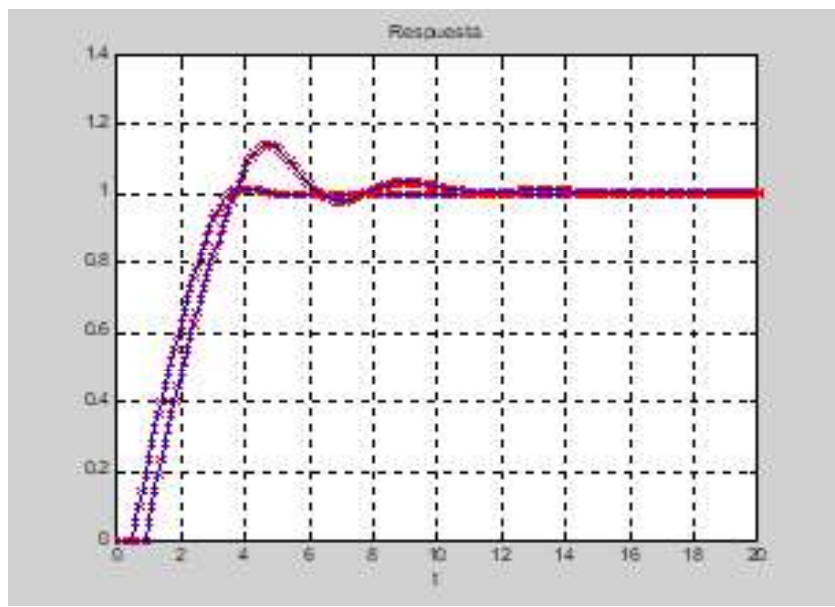

Fig. 8. Respuesta ante variaciones en el tiempo de retardo.

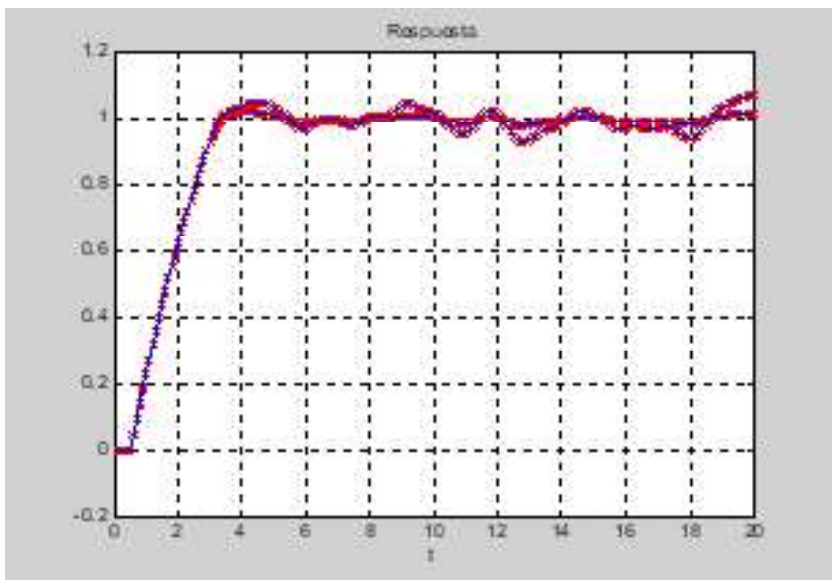

Fig. 9. Respuesta con ruido.

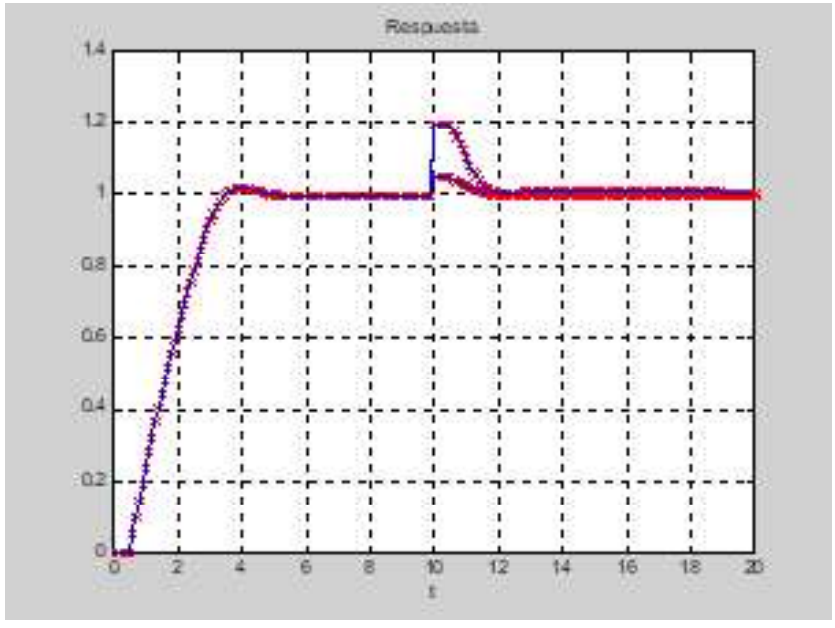

Fig. 10. Respuesta ante perturbaciones de carga (Turbina de gas fuera de línea). nuo) y el controlador digital neurodifuso (trazo estrellado). En cada figura se muestran dos casos de respuesta típicos, los cuales demuestran la equivalencia del comportamiento del lazo de control usando ambos controladores.

En las figuras 11, 12 y 13 se muestra, en forma resumida, el desempeño IAE del controlador cuando los parámetros del modelo: ganancia en estado estable, tiempo de retardo y constante de tiempo respectivamente, se hicieron variar en un rango que va desde el $50 \%$ hasta el $200 \%$ de su valor nominal. Como puede observarse, el desempeño en la vecindad de los valores nominales solamente presenta una variación pequeña, lo cual se espera en una aplicación real.

Las figuras 14 y 15 presentan el desempeño del lazo de control sujeto a perturbaciones aditivas aleatorias en la señal de control $u$ y en la señal de salida de la planta $y$, respectivamente. Nuevamente el desempeño del controlador presenta variaciones suaves en un gran rango. La Fig. 16 muestra el desempeño ante un cambio de carga en la señal de salida de la

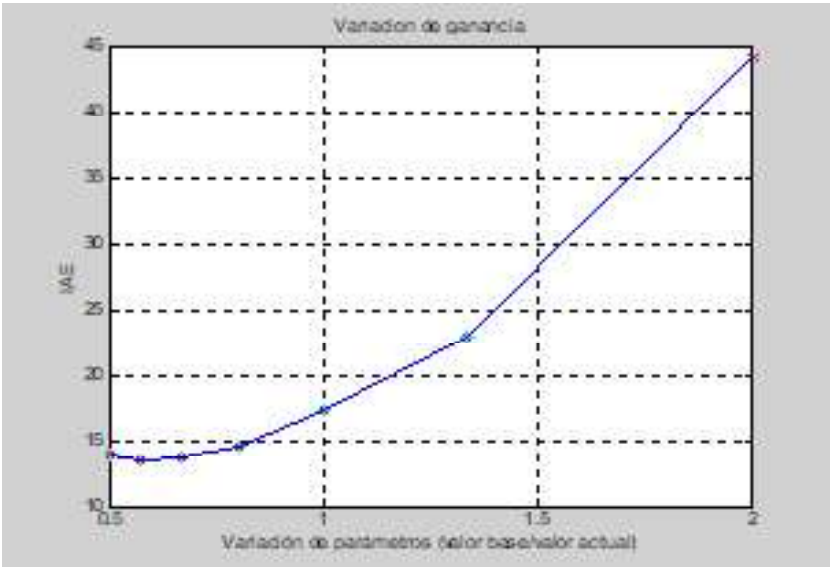

Fig. 11. Desempeño ante variaciones en la ganancia.

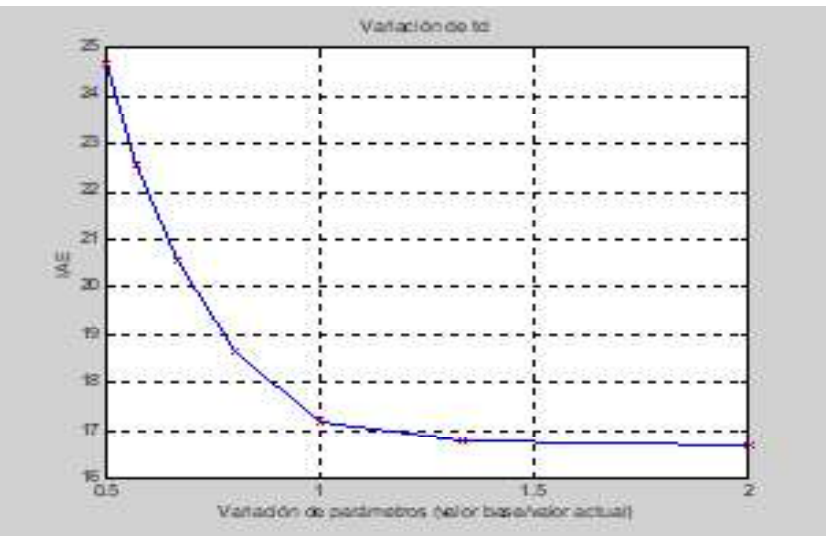

Fig. 12. Desempeño ante variaciones en el tiempo de retardo. 


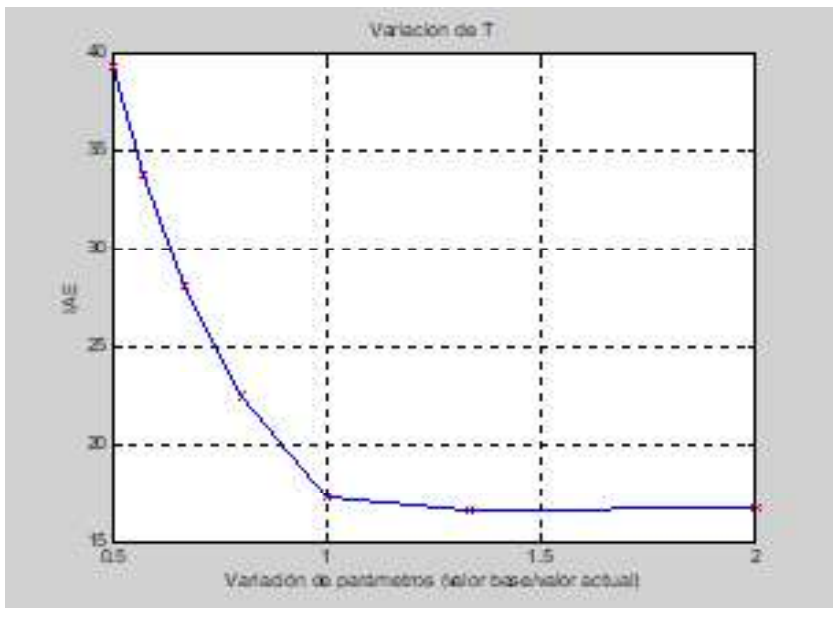

Fig. 13. Desempeño ante variaciones en la constante de tiempo.

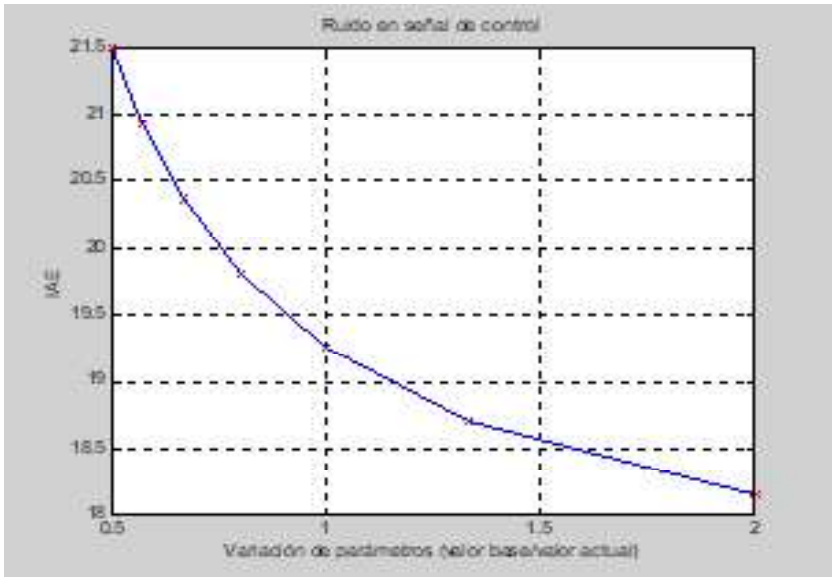

Fig. 14. Desempeño ante ruido en la señal de control.

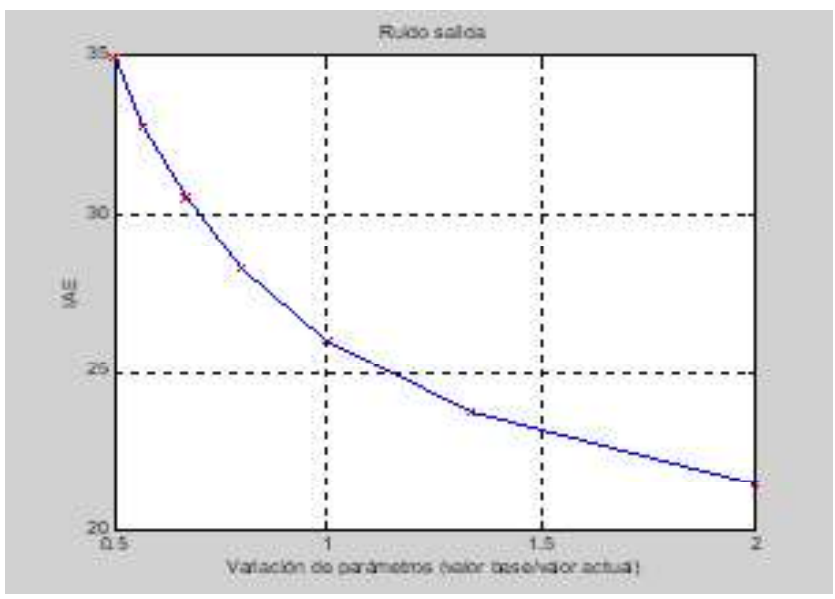

Fig. 15. Desempeño ante ruido en la salida del proceso.

planta, simulado mediante la adición de un escalón a los 10 segundos del inicio de la simulación. Con estos resultados se

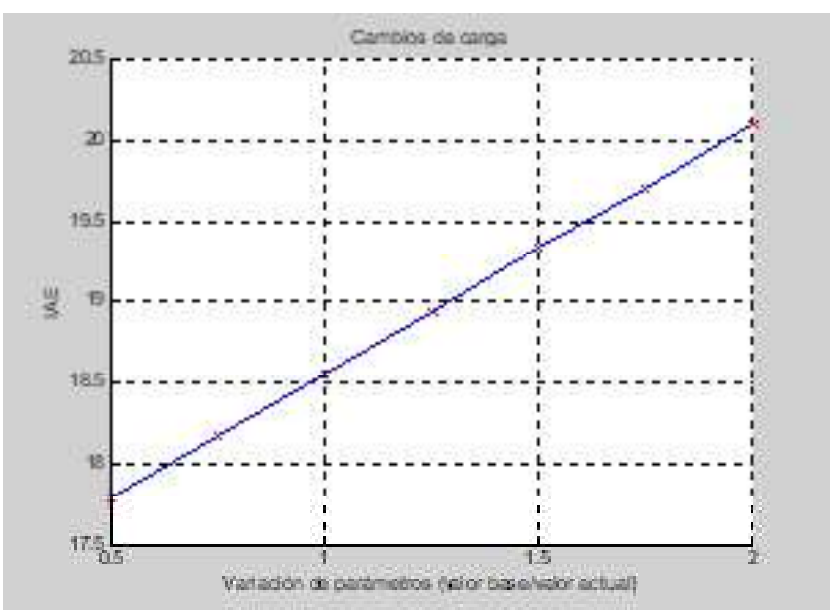

Fig. 16. Desempeño ante cambios de carga a la salida.

observa que el controlador rechaza perturbaciones, tal y como se espera en una aplicación real.

\section{Conclusiones}

En este trabajo se presentó un controlador neurodifuso que emula la dinámica de un controlador PI convencional en configuración de velocidad. El sistema de inferencia difuso integrado en el controlador es del tipo TSK de primer orden e implementa un mapeo $R^{2} \rightarrow R$ de la señal de error y su razón de cambio a la diferencial de la señal de control. El diseño del sistema de inferencia difuso se lleva a cabo fuera de línea empleando el método ANFIS con patrones de aprendizaje obtenidos de las entradas y la salida de un controlador PI convencional.

Los resultados obtenidos en las pruebas de análisis de estabilidad del controlador muestran que el controlador neurodifuso digital posee en su desempeño la factibilidad de emplearlo en el mismo tipo de aplicaciones que un controlador PI convencional mediante una sustitución directa.

Después de la demostración de factibilidad del controlador neurodifuso, reportada en este artículo, se planea su uso para el control de la velocidad, durante el arranque y la potencia generada en una unidad turbogás, mejorando su rendimiento bajo índices de desempeño específicos.

\section{Agradecimientos}

Este trabajo fue realizado en el Instituto Nacional de Electricidad y Energías Limpias (INEEL), con el apoyo de la Sección de Estudios de Posgrado e Investigación de la Escuela Superior de Ingeniería Mecánica y Eléctrica Zacatenco. 


\section{Referencias}

[1] A. Giampaolo, Gas turbine handbook: principles and practices, The Fairmont Press, $3^{\mathrm{a}}$ ed., Londres, 2006.

[2] G. Rogers, H. Saravanamutto, Gas turbine theory. Longman House, $4^{\mathrm{a}}$ ed., Londres, 1996.

[3] General Electric, Turbina de gas (01), 2019. Recuperado de https://www.ge.com/power/gas/gas-turbines

R. Garduño, M. Sánchez, "Control System Modernization:

[4] Turbogás Unit Case Study," IFAC Symposium on Control of Power Plants and Power Systems, vol. 2, pp. 245-250, Cancún, México 1995.

R. Garduño, G. Madrigal, M. Sánchez, "Modernización de

[5] los Sistemas de Control en la Central Termoeléctrica de Ciclo Combinado Gómez Palacio, parte III: Turbinas de Gas". Boletín IIE, vol. 20, no. 3, pp. 117-125, mayo-junio 1996.

J. Reason, "Why High Performance Controls are Vital for

[6] Gas Turbines," Electrical World, pp. 71-74, abril 1989.

N. Hadroug, A. Hafaifa, M. Guemana, "Heavy duty gas

[7] turbine monitoring based on adaptive neuro-fuzzy inference system: speed and exhaust temperature control", Mathematics-in-Industry Case Studies, vol.8, no.8, 2017, https://doi.org/10.1186/s40929-017-0017-8

A. Rodriguez-Martinez, R. Garduno-Ramirez, L. G. Vela-

[8] Valdes, "PIFuzzy Gain-Scheduling Speed Control at Startup of a Gas-Turbine Power Plant", IEEE Transactions On Energy Conversion, vol. 26, no. 1, 2011.

S. De Lara, R. Garduño, M. Sánchez, L. Castelo, M.

[9] Carretero, "A Process Knowledge-Based Controller for Maneuverability Improvement of a Nonlinear Industrial Process," Springer, vol. 25-27, noviembre 2002.
[10] C. D. García, "Control Digital de Unidades Turbogás", Tesis de Maestría, Centro Nacional de Investigación y Desarrollo Tecnológico, México, 1997.

[11] C. D. García, R. Garduño, "Gas Turbine Fuzzy Speed Control", 2nd Joint Mexico-USA International Conference on Neural Networks and Neurocontrol, Sianka'an, Mexico 1997.

[12] C. D. García, R. Garduño, "Control Software System of a Turbogás Power Unit", 5th IFAC Workshop on Algorithms and Architectures for Real Time Control, pp. 131-136, Cancún, México, 1998.

[13] M. Sánchez, L. Bahamaca, "Realización de controladores lógicos difusos para el control de procesos en tiempo real: especificación y diseño", $2^{\circ}$ Encuentro Nacional de Computación, Pachuca, México,1999.

[14] M. Sánchez, "Control for Gas Turbines", 1st Annual ISA POWID/EPRI/DOE Joint Controls and Instrumentation Conference, San Antonio, USA 2000.

[15] M. Pat, S. de Lara, "Sintonización con Redes Neuronales de un Controlador PID. Aplicación a una unidad turbogás", Información Tecnológica, vol. 10, no. 6, pp. 25-32, 1999.

[16] S. De Lara, M. A. Martínez," Desarrollo del Sistema para la Sintonización de Controladores Difusos Usando Redes Neuronales Artificiales," IIE reporte interno: 11500INDT (A5), enero-marzo 1999.

[17] J.S.R Jang, N. Gulley, Fuzzy Logic Toolbox, Natick, MS, Mathworks 2014.

[18] J. Ramírez, M. Chacón, "Redes neuronales para el procesamiento de imágenes, una revisión de la última década", RIEE\&C, vol. 9, no. 1, 2011.

[19] P. Ponce, Inteligencia Artificial con Aplicaciones a la Ingeniería, Alfaomega, 2010. 
